\title{
ORÇAMENTO: PERSPECTIVAS TEÓRICAS DAS PESQUISAS CIENTÍFICAS INTERNACIONAIS
}

\section{BUDGET: THE THEORETICAL PERSPECTIVES OF INTERNATIONAL SCIENTIFIC RESEARCH}

0 artigo foi aprovado e apresentado no evento: $4^{\circ}$ Congresso de Gestão e Controladoria da Unochapecó, $2^{\circ}$ Congresso FURB de Ciências Contábeis, International Conference in Management and Accounting, $4^{\circ}$ Congresso de Iniciação Científica, realizado de 17/10/2019-19/10/2019, na UnoChapecó, Chapecó, Santa Catarina, Brasil.

\section{RESUMO}

O orçamento pode aprimorar a gestão financeira por meio do planejamento e controle dos recursos financeiros. Embora extensivamente pesquisado em contabilidade gerencial, no âmbito do setor público ainda carece de estudos acerca dos avanços científicos. Assim, este artigo objetiva identificar as características dos artigos científicos internacionais sobre orçamento público e classificar esses artigos de acordo com as perspectivas teóricas de Covaleski et al. (2007): econômica, psicológica e sociológica. Trata-se de um estudo sistemático de revisão da literatura, por meio da seleção e análise bibliográfica de artigos científicos internacionais com base na ferramenta Knowledge Development Process-Constructivist (Proknow-C). A partir da análise do Portfólio, composto por 33 artigos, foram encontrados os seguintes resultados: (i) não há concentração regional dos artigos; (ii) a maioria dos artigos utiliza metodologia qualitativa; (iii) os artigos utilizam principalmente as perspectivas econômica e sociológica, isoladamente, em suas análises; (iv) dois artigos estabeleceram uma integração entre as três perspectivas teóricas. Por fim, reafirma-se o entendimento de Covaleski et al. (2007), de que o sistema orçamentário será melhor compreendido a partir da integração das perspectivas econômica, sociológica e psicológica.

Palavras-chave: Orçamento. Setor Público. Economia. Psicologia. Sociologia.

\begin{abstract}
The budget can improve financial management through planning and control of financial resources. Although extensively researched in managerial accounting, within the public sector still lacks studies on scientific advances. Thus, this article aims to identify the characteristics of international scientific articles on public budget and to classify these articles according to theoretical perspectives of Covaleski et al. (2007): economic, psychological and sociological. This is a systematic review of the literature, through the selection and bibliographic analysis of international scientific articles based on the Knowledge Development Process-Constructivist (Proknow-C). Starting the analysis of the Portfolio, composed of 33 articles, the following results were found: (i) the articles are distributed around the world; (ii) most articles use qualitative methodology; (iii) most articles use the economic and sociological perspectives alone in their analyzes; (iv) two articles established an integration between the three theoretical perspectives. Finally, it was found that the understanding of Covaleski et al. (2007) still persists, that the budget system will be better understood from the integration of economic, sociological and psychological perspectives.
\end{abstract}

Keywords: Budget. Public sector. Economy. Psychology. Sociology.

\author{
Paula Cristina Leite Guesser \\ Mestranda em Contabilidade pela \\ Universidade Federal de Santa Catarina \\ (UFSC). Especialista em Contabilidade \\ Pública pela Universidade do Sul de \\ Santa Catarina (UNISUL). Graduada em \\ Ciências Contábeis pela UFSC. Graduada \\ em Geografia pela Universidade do \\ Estado de Santa Catarina (UDESC) \\ Analista Judiciário, especialidade \\ Contabilidade no Tribunal Regional \\ do Trabalho da $12^{\text {a }}$ Região (TRT12). \\ Contato: Rua da Amizade, 78, Centro, \\ Antônio Carlos, SC, CEP 88.180-000. \\ E-mail: paulalguesser@gmail.com
}

\section{Sérgio Murilo Petri}

Doutor em Engenharia de Produção e Sistemas (UFSC). Mestre em Engenharia de Produção e Sistema (UFSC). Graduado em Ciências Contábeis pela Universidade do Vale do Itajaí (UNIVALI). Docente do curso de Ciências Contábeis na Universidade Federal de Santa Catarina (UFSC). Contato: Rua João Meirelles, 1435, Apto 101, Bloco B, Florianópolis, SC, CEP: 88.085-201. E-mail: smpetri@gmail.com ou sergio. petri@ufsc.br

\footnotetext{
Carlos Eduardo Facin Lavarda

Doutor em Contabilidade pela Universitat de Valencia, Espanha. Bolsista CNPq. Docente do Curso de Graduação em Ciências Contábeis e do Programa de Pós-graduação em Contabilidade na Universidade Federa de Santa Catarina (UFSC). Contato: R Eng. Agronômico Andrei Cristian Ferreira, $\mathrm{s} / \mathrm{n}$ - Trindade, Florianópolis, SC, CEP: 88040-900. E-mail: eduardo. lavarda@ufsc.br
} 


\section{INTRODUÇÃO}

O orçamento é um dos tópicos mais extensivamente pesquisados em contabilidade gerencial (Covaleski, Evans III, Luft, \& Shields, 2007, p. 587). E as pesquisas internacionais sobre orçamento enfatizam um maior entendimento sobre o seu papel dentro do contexto organizacional (Silva \& Lavarda, 2014), a partir das perspectivas teóricas da economia, psicologia e sociologia (Covaleski et al., 2007, p. 587).

Por isso, este artigo fundamenta-se no estudo de Covaleski et al. (2007), uma vez que ele apresenta uma proposta mais abrangente acerca de como as organizações são compostas e moldadas a partir do orçamento. Isto é, o orçamento não é visto apenas como um instrumento elaborado com base nos interesses dos indivíduos ligados à organização, mas também como um instrumento que impõe regras capazes de moldar a cultura organizacional.

Todavia, a área de orçamento carece de trabalhos que avaliam a produção científica e investigam os tópicos abordados e metodologias aplicadas, de forma que estudar as características descritas na literatura é importante para se conhecer o nível de desenvolvimento e nível de inovação do tema (Lunkes, Feliu \& Rosa, 2011). São raros tais estudos em âmbito nacional e internacional (Lunkes et al., 2011, p. 114). No Brasil, podem se destacar, por exemplo, os estudos de Leite, Cherobim, Silva e Bufrem (2008), Lunkes et al. (2011) e Silva e Lavarda (2014), todos acerca da produção científica sobre orçamento empresarial.

Percebe-se assim, que pesquisas de revisões de literatura em contabilidade sobre o controle orçamentário mostram um domínio quase completo da pesquisa sobre as organizações do setor privado (Chapman, 1997, Hartmann, 2000, Chenhall, 2003 como citado em Johansson \& Siverbo, 2014, p. 1).Portanto, identificou-se como lacuna de pesquisa a necessidade de ampliar a produção científica acerca da literatura sobre orçamento no setor público. Para tanto, optou-se por coletar artigos científicos internacionais sobre o tema nos últimos anos, já que eles aparecem em maior quantidade nos periódicos internacionais do que nos periódicos nacionais (Silva \& Lavarda, 2014).

Propõe-se, dessa forma, identificar as características dos artigos selecionados e classificar esses estudos de acordo com as perspectivas teóricas segundo Covaleski et al. (2007): econômica, psicológica e sociológica. Pois essas perspectivas ajudam a entender como ocorrem as relações entre as instituições e as pessoas que moldam e são moldadas pelo processo orçamentário no âmbito público.

Considerando que o setor público vem passando por recentes reformas, entre elas a busca da melhoria do seu desempenho, por meio da adoção de práticas do setor privado (Abu-Doleh \& Weir, 2007; Conway, Fu, Monks, Alfes \& Bailey, 2016; Tanwir \& Chaudhry, 2016), é possível que a maior parte das pesquisas sobre orçamento adote uma perspectiva econômica. Por outro lado, a pesquisa sobre uma perspectiva psicológica se tornou muito importante nos últimos anos, tendo em vista que muitas organizações públicas vêm enfrentando mudanças e cortes orçamentários, o que impacta diretamente nas atitudes e comportamentos dos seus funcionários (Kiefer, Hartley, Conway, \& Briner, 2014).

Ainda por outro ângulo, é provável que a perspectiva sociológica acerca do orçamento seja particularmente adotada nos estudos do setor público, em especial reconhecendo as lógicas institucionais inerentes ao Governo, como exemplificado por Hyvönen, Järvinen, Pellinen, \& Rahko (2009), pois o orçamento público visa ao alcance do bem-estar social (Johansson \& Siverbo, 2014), diferentemente das organizações privadas que buscam aumentar a sua lucratividade.

Assim, esta pesquisa busca evidenciar, em especial, quais as perspectivas estão mais presentes nas pesquisas internacionais empíricas de contabilidade gerencial e administração, e de que forma elas estão sendo abordadas, por meio da identificação de variáveis orçamentárias e não-orçamentárias, como por exemplo, orçamento participativo, avaliação de desempenho baseada no orçamento, processo orçamentário, mobilização de poder, desempenho individual e gerencial.

Como justificativa do estudo, tem-se então, a necessidade de verificar o avanço científico sobre orçamento público em relação às perspectivas teóricas delineadas por Covaleski et al. (2007), no intuito de contribuir com um maior entendimento sobre o processo de elaboração e execução orçamentária. Ante o exposto, a estrutura deste estudo é composta por esta Introdução; pelo Referencial Teórico; pela Metodologia; pelos Resultados; pelas Considerações finais; e, por fim, pelas Referências.

\section{REFERENCIAL TEÓRICO}

\subsection{Orçamento}

O orçamento tem a função de converter os objetivos estratégicos em metas e valores operacionais, gerando um guia para as operações e decisões dos gestores (Lunkes et al., 2011, p. 114). Em outras palavras, o orçamento pode aumentar a eficiência por meio do planejamento e da coordenação e apoiar o controle e o aprendizado por meio da comparação dos resultados reais com os planos (Covaleski et al., 2007).

Os orçamentos são instrumentos de governança que podem ser usados para transmitir os objetivos organizacionais aos atores da organização (Abernethy \& Brownell, 1999; Covaleski et al., 2003; Goddard, 2004 como citado em Mutiganda, 2016, p. 1). De forma semelhante ao setor privado, no setor público o orçamento envolve o planejamento, o controle e a prestação de contas, para garantir que os recursos sejam alocados em prol dos propósitos políticos de bem-estar dos cidadãos (Johansson \& Siverbo, 2014). Portanto, o orçamento público envolve a comunicação e implementação das políticas públicas aos funcionários (Kiefer et al., 2014), podendo servir para institucionalizar políticas que não estão 
necessariamente relacionadas com a maneira com que os atores da organização pensam e agem (Fallan et al., 2010, Lapsley et al., 2011 como citado em Mutiganda, 2016).

O orçamento é uma prática permanente e importante na formação de relações econômicas e políticas dentro e entre organizações (Covaleski, Dirsmith, \& Weiss, 2013) e está sempre em constante mudança. No setor público, essas mudanças estão relacionadas à Nova Gestão Pública e ao gerencialismo das últimas três décadas, na qual a contabilidade passou a utilizar o regime de competência ao invés do regime de caixa (Becker, Jagalla, \& Skærbæk, 2014) para melhorar a eficiência das organizações.

Portanto, tanto fatores internos quanto externos impactam no processo orçamentário das organizações, o que pode ser explicado com maior profundidade a partir das perspectivas teóricas do orçamento. Mas, ressalta-se que a nível mundial, o orçamento é uma exigência legal, que por si só já impõe uma característica institucional forte. O estudo de Bradbury e Crain (2001, p. 322), por exemplo, apontou que a separação do poder legislativo em duas câmaras exerce um impacto considerável sobre a política fiscal.

\subsection{Perspectivas teóricas do orçamento}

Segundo Covaleski et al. (2007), o orçamento é estudado a partir das perspectivas teóricas da economia, psicologia e sociologia. A Tabela 1 evidencia as semelhanças e diferenças das variáveis orçamentárias e não orçamentárias de cada uma dessas perspectivas.

Tabela 1 - Perspectivas teóricas do orçamento

\begin{tabular}{|c|c|c|c|}
\hline & Economia & Psicologia & Sociologia \\
\hline $\begin{array}{l}\text { Variáveis } \\
\text { orçamentárias }\end{array}$ & $\begin{array}{l}\text { Características das } \\
\text { práticas de orçamento e } \\
\text { remuneração, incluindo } \\
\text { contratos baseados em } \\
\text { desempenho, orçamento } \\
\text { participativo, orçamento } \\
\text { de capital e investigação } \\
\text { de variação. }\end{array}$ & $\begin{array}{l}\text { Orçamento participativo, } \\
\text { dificuldade na meta orçamentária, } \\
\text { ênfase no orçamento na avaliação } \\
\text { de desempenho, remuneração } \\
\text { baseada no desempenho. }\end{array}$ & $\begin{array}{l}\text { 1. Teoria da Contingência: orçamento } \\
\text { participativo, orçamento com } \\
\text { base no desempenho, importância } \\
\text { orçamentária, uso de orçamentos } \\
\text { operacionais para controle gerencial. } \\
\text { 2. Teoria institucional: processo } \\
\text { orçamentário. }\end{array}$ \\
\hline $\begin{array}{l}\text { Variáveis não } \\
\text { orçamentárias }\end{array}$ & $\begin{array}{l}\text { 1. Mercado de trabalho: } \\
\text { habilidade e preferências } \\
\text { dos funcionários; } \\
\text { 2. Estrutura da informação: } \\
\text { informações públicas e } \\
\text { privadas, incerteza do } \\
\text { estado; } \\
\text { 3. Resultados: bem-estar } \\
\text { individual, desempenho } \\
\text { organizacional, folga } \\
\text { orçamentária. }\end{array}$ & $\begin{array}{l}\text { 1. Estados mentais: atitudes, } \\
\text { motivação, satisfação, estresse; } \\
\text { 2. Contexto organizacional: } \\
\text { incerteza de tarefa; } \\
\text { 3. Comportamento: jogo; } \\
\text { 4. Desempenho: individual e } \\
\text { gerencial. }\end{array}$ & $\begin{array}{l}\text { 1. Teoria da Contingência: tamanho } \\
\text { organizacional, estruturação } \\
\text { de atividades, descentralização, } \\
\text { tecnologia automação, subunidade } \\
\text { interdependência, diversificação } \\
\text { estratégica. } \\
\text { 2. Teoria institucional: valor simbólico } \\
\text { da contabilidade, negociação de } \\
\text { recursos e de barganha, escondendo } \\
\text { e mobilizando poder, mudança } \\
\text { ambiental e mudança organizacional. }\end{array}$ \\
\hline
\end{tabular}

Nota. Fonte: Recuperado de "Budgeting research: three theoretical perspectives and criteria for selective integration" de M. A. Covaleski, J. H. Evans III, J. L. Luft, M. D. Shields, 2007, Handbooks of management accounting research, 2(1), 591.

Como se percebe a partir da Tabela 1, a perspectiva econômica foca no resultado organizacional, fruto da eficiência da prestação dos serviços e da relação entre principal e agente no processo orçamentário. A perspectiva psicológica volta-se para o interior do indivíduo e para os comportamentos desses funcionários em relação ao orçamento adotado. Enquanto a perspectiva sociológica centra-se no aspecto coletivo da construção e execução do orçamento, derivado das relações institucionais e de poder dos atores políticos. As subseções seguintes detalham um pouco mais essas perspectivas.

Na pesquisa baseada em economia, o estudo concentra-se na natureza das compensações ótimas nos contratos de trabalho entre proprietários e funcionários com preferências conflitantes e assimetria de informações, e como essas compensações afetam o desempenho organizacional (Covaleski et al., 2007). A Teoria da Agência explica como se dá a relação de contrato entre principal e agente, onde estes acordam objetivos em comum em troca de compensação. 
É uma teoria puramente econômica e racional, que entende que o funcionário está satisfeito com a remuneração e o gestor com o serviço prestado (Covaleski et al., 2007). Por exemplo, o fato de muitos políticos não usarem informações de desempenho pode também ser considerada como um comportamento economicamente racional, já que eles se concentram apenas nas informações relevantes para seus interesses próprios (Ter Bogt, Van Helden \& Van Der Kolk, 2015).

A pesquisa baseada na psicologia estuda a natureza das reações dos indivíduos às práticas orçamentárias, envolvendo variáveis como orçamento participativo, comportamento dos funcionários, desempenho individual e remuneração com base no orçamento (Covaleski et al., 2007). Parte-se do entendimento de que a melhoria do desempenho individual produz a melhoria do desempenho organizacional como um todo.

Em geral, o orçamento participativo aumenta o engajamento dos funcionários, porque eles se sentem importantes para a instituição. Por outro lado, reduções de recursos no setor público, tem efeito negativo sobre os funcionários em termos de menor satisfação e motivação no trabalho e maior frustação sobre ser ou não capaz de fornecer um serviço adequado (Cameron, Whetten, \& Kim, 1987; Levine, 1979; Yang \& Kassekert, 2009 como citado em Kiefer et al., 2014, p. 5).

Quanto à pesquisa baseada na sociologia, o foco está no papel do orçamento nos processos organizacionais e seus resultados (Covaleski et al., 2007). A Teoria da Contingência e Institucional são utilizadas para compreender como se constituem e se transformam as relações políticas dentro e entre as organizações e o ambiente em que estão inseridas. A Teoria da Contingência preceitua que a organização tem uma natureza sistêmica e compartilha uma tradição com perspectivas econômicas e psicológicas sobre orçamento ao investigar influências na eficiência da tomada de decisão organizacional e no alinhamento do indivíduo com os objetivos organizacionais (Covaleski et al., 2007, p. 607). A Teoria Institucional se concentra explicitamente nos papéis simbólicos das ferramentas de tomada de decisões racionais na organização, como os orçamentos, e na maneira como esse simbolismo promove o poder e o interesse nos processos de tomada de decisão (Covaleski et al., p. 607).

O processo orçamentário no setor público tem sido reconhecido como um contexto altamente institucionalizado, em que a natureza dessas organizações é intimamente ligada com as suas práticas orçamentárias (Hopwood, 1984, Wildavsky, 1964 como citado em Ahrens \& Ferry, 2018, p. 12). Acerca desse processo de institucionalização, Burns e Scapens (2000) esclarecem que a reprodução de ações pelos atores é responsável por criar novas rotinas, que passam a ser institucionalizadas e moldam o processo de mudança das organizações. Já na Teoria Neoinstitucional as organizações respondem às pressões externas decorrentes dos seus ambientes institucionais, independentemente de haver ou não aumento da eficiência e eficácia da organização ao adotar tais mudanças, pois o que importa é a conformidade dessas respostas em termos de legitimidade social dentro do ambiente institucional (Cohen \& Karatzimas, 2014).

\section{METODOLOGIA}

Esta seção trata do enquadramento metodológico, do procedimento para a coleta dos dados e do procedimento para análise dos dados e classificação dos artigos. O enquadramento metodológico refere-se à caracterização do tipo de abordagem adotada na pesquisa. No presente estudo optou-se por utilizar uma visão de conhecimento construtivista, entendida esta como uma abordagem mais próxima do interpretativismo, que visa construir conhecimento sobre a realidade. A natureza do objetivo desta pesquisa é descritiva e a análise é qualitativa, já que a pesquisa é caracterizada como a tentativa de compreensão dos significados e características da situação (Richardson, 2008).

Neste caso, este artigo visa analisar um Portfólio Bibliográfico (PB), representativo de um fragmento da literatura, por meio da interpretação dos autores para gerar conhecimento sobre o tema orçamento. Essa interpretação é tida como fonte primária da pesquisa, enquanto os artigos selecionados são tidos como fonte secundária da pesquisa (Richardson, 2008).

O instrumento de intervenção utilizado para realizar a revisão sistemática da literatura é o Knowledge Development Process-Constructivist (ProKnow-C), cuja operacionalização nesta pesquisa será realizada por meio das etapas: Seleção do Portfólio Bibliográfico (PB), Análise Bibliométrica e Pergunta de Pesquisa (Valmorbida \& Ensslin, 2017). A seleção do PB tem início com a definição das palavras-chave que dão origem ao comando de busca a ser aplicado na base de dados da CAPES. Nesta pesquisa o comando de busca utilizado na Web of Science foi o seguinte: (budget*) AND ("public sector" OR "public administration” OR “government”).

Aplicaram-se, ainda, como filtros a seleção de artigos publicados na língua inglês e em revistas de Business, management and accounting no período de 01.01.2013 a 17.01.2019, uma vez que este período representa as pesquisas mais recentes. A Figura 1 demonstra com mais detalhes os passos adotados para filtragem dos 657 artigos encontrados na busca na base de dados realizada em 17.01.2019. 
Figura 1. Filtragem do Portfólio Bruto

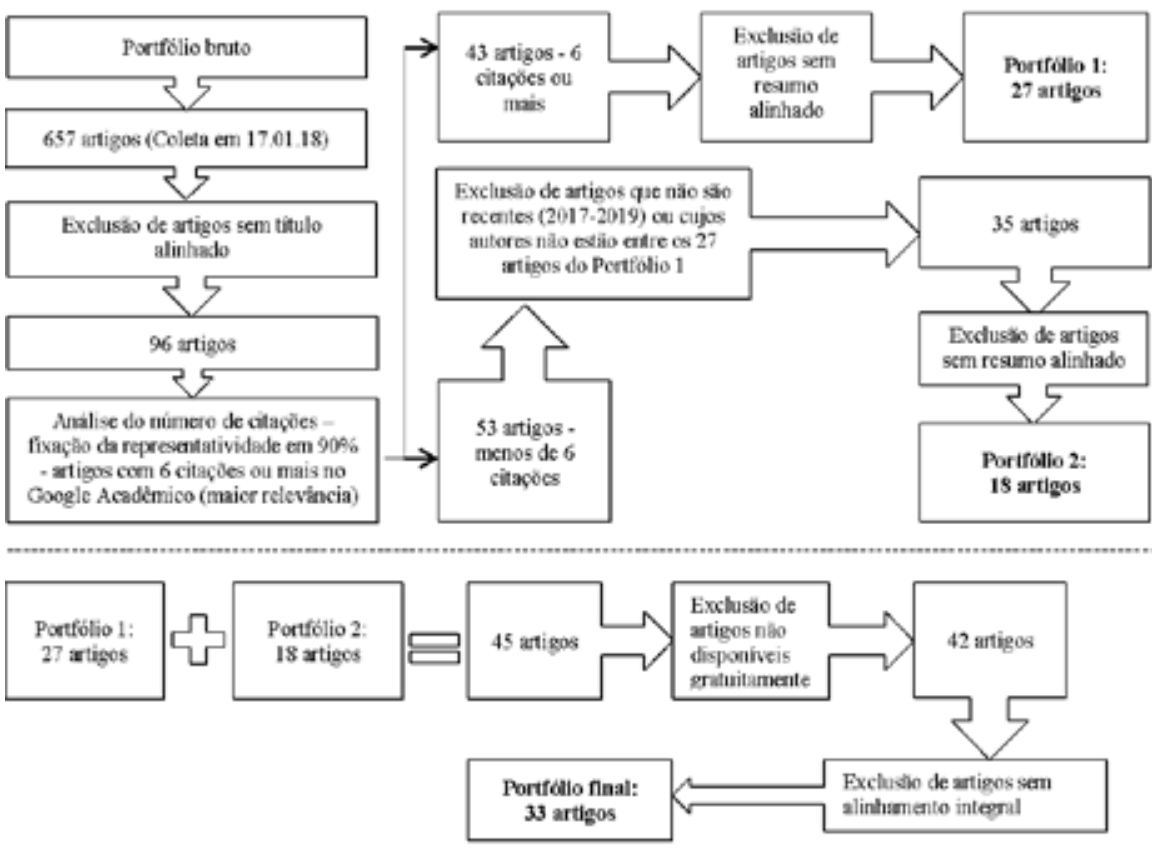

Fonte: Elaborada pelos autores com base na etapa de Filtragem do ProKnow-C.

Conforme a Figura 1, a filtragem teve início com a exclusão de artigos sem alinhamento, isto é, apenas pela leitura do título foi possível verificar os artigos que realmente tratavam de orçamento no setor público. A seguir, os artigos que tiveram maior relevância (6 citações ou mais - que representavam $90 \%$ dos artigos) foram separados dos demais. Aqueles de maior relevância tiveram seus resumos analisados e permaneceram apenas aqueles que tratavam do tema, gerando o Portfólio 1. Já em relação aos artigos que tiveram menos de 6 citações, houve uma repescagem daqueles mais recentes (01.01.2017-17.01.2019) e cujos autores estão no rol de autores do portfólio 1; dos quais permaneceram aqueles que tinham o resumo alinhado, gerando o Portfólio 2. A junção do Portfólio 1 e 2 gerou 45 artigos, sendo 42 disponíveis gratuitamente. A análise integral desses 42 artigos revelou que apenas 33 artigos apresentavam o orçamento no setor público com referência clara às características econômicas, sociológicas ou psicológicas que compõem a formação e as mudanças orçamentárias.

Com base nos procedimentos do ProKnow-C, foi possível selecionar 33 artigos empíricos disponíveis online que compõem o Portfólio Bibliográfico (PB), o qual será analisado na presente pesquisa. Esses artigos estão listados na seção das Referências com um número sequencial de 1 até 33, sinalizados com colchetes ao final da citação.

O procedimento para análise dos dados na etapa da Análise Bibliométrica abrange, em um primeiro momento, a verificação de três aspectos gerais: localização das pesquisas, metodologias aplicadas e evolução temporal. Em um segundo momento, o objetivo é classificar os artigos conforme as perspectivas teóricas segundo Covaleski, et al. (2007), descritas na Seção 2.2, por meio da identificação das variáveis orçamentárias e não-orçamentárias que são tratadas nos artigos do PB. Portanto, por meio de um fragmento da literatura, pretende-se analisar se as pesquisas orçamentárias no setor público abordam variáveis enquadradas nas perspectivas econômica, psicológica e sociológica.

\section{RESULTADOS}

\subsection{Aspectos gerais das pesquisas}

Seguindo a ordem descrita na metodologia, primeiramente, são situadas as pesquisas internacionais sobre o assunto e ao mesmo tempo elas já aparecem categorizadas na Tabela 2 conforme as perspectivas teóricas de Covaleski, et al. (2007): 
Tabela 2 - Localização das pesquisas internacionais

\begin{tabular}{|c|c|c|}
\hline Perspectiva & Localização & Artigo (numeração 1-33) \\
\hline Econômica & $\begin{array}{l}\text { Nigéria } \\
11 \text { países da Ásia } \\
\text { Arábia Saudita } \\
\text { Taiwan } \\
\text { Indonésia } \\
\text { Reino Unido, Austrália e Nova Zelândia } \\
\text { Dinamarca } \\
\text { Estados Unidos }\end{array}$ & $\begin{array}{l}23 \\
27 \\
21 \\
20 \\
28 \\
12 \\
30 \\
4,17,31\end{array}$ \\
\hline Sociológica & $\begin{array}{l}\text { Sri Lanka } \\
\text { Austrália } \\
\text { Europa - Comissão Europeia } \\
\text { Itália } \\
\text { Finlândia } \\
\text { Suécia } \\
\text { Grécia } \\
\text { Estados Unidos }\end{array}$ & $\begin{array}{l}13 \\
33 \\
11 \\
26,29 \\
3 \\
8 \\
16 \\
14\end{array}$ \\
\hline Psicológica & $\begin{array}{l}\text { Israel } \\
\text { Austrália } \\
\text { Alemanha } \\
\text { Reino Unido }\end{array}$ & $\begin{array}{l}19 \\
25 \\
5 \\
1,2\end{array}$ \\
\hline Sociológica e Econômica & $\begin{array}{l}\text { Sri Lanka } \\
\text { Holanda } \\
10 \text { municípios da Europa } \\
\text { Estados Unidos }\end{array}$ & $\begin{array}{l}18 \\
7 \\
15 \\
22\end{array}$ \\
\hline $\begin{array}{l}\text { Sociológica e Psicológica } \\
\text { Sociológica, Econômica e Psicológica }\end{array}$ & $\begin{array}{l}\text { Israel } \\
\text { Finlândia } \\
\text { Alemanha e Itália } \\
\text { Suécia } \\
\text { Estados Unidos }\end{array}$ & $\begin{array}{l}10 \\
32 \\
9 \\
6 \\
24\end{array}$ \\
\hline
\end{tabular}

Nota. Fonte: Elaborada pelos autores com base na etapa de Filtragem do ProKnow-C.

Como demonstrado na Tabela 2, as pesquisas sobre orçamento público estão sendo desenvolvidas em diversos países do mundo. Todavia, não foi possível identificar relação entre as perspectivas teóricas adotadas nas pesquisas com a localização.

Em relação à metodologia aplicada a maior parte dos artigos (vinte e três) utilizaram uma abordagem qualitativa, em especial por meio de análise de documentos e entrevistas com funcionários das instituições públicas. Enquanto os demais artigos (dez) utilizaram uma abordagem quantitativa, com aplicação de métodos estatísticos.

As pesquisas com abordagem quantitativa são bem recentes, publicadas a partir de 2016. O que pode sugerir que os estudos estão se encaminhando para a análise estatística de variáveis relacionadas ao orçamento público. Mas, até o momento as pesquisas com abordagem qualitativa ainda dominam esse campo de pesquisa, o que pode estar ligado ao fato de que o orçamento no setor público envolve um ambiente de múltiplas variáveis, cuja complexidade dificulta a sua mensuração. 
Nota-se também, que há uma predominância pela adoção das perspectivas econômica e sociológica nos estudos orçamentários do setor público, tanto em relação ao período de análise (2013-2019), quanto em relação aos anos mais recentes (2018-2019). A Seção 4.2 pretende aprofundar a análise acerca das perspectivas teóricas adotadas.

\subsection{Perspectivas teóricas das pesquisas}

A Figura 2 ilustra a quantidade de artigos publicados classificados por perspectiva teórica adotada, a partir da descrição das variáveis orçamentárias e não orçamentárias de Covaleski, et al. (2007).

Figura 2. - Quantidade de artigos por perspectiva teórica adotada.

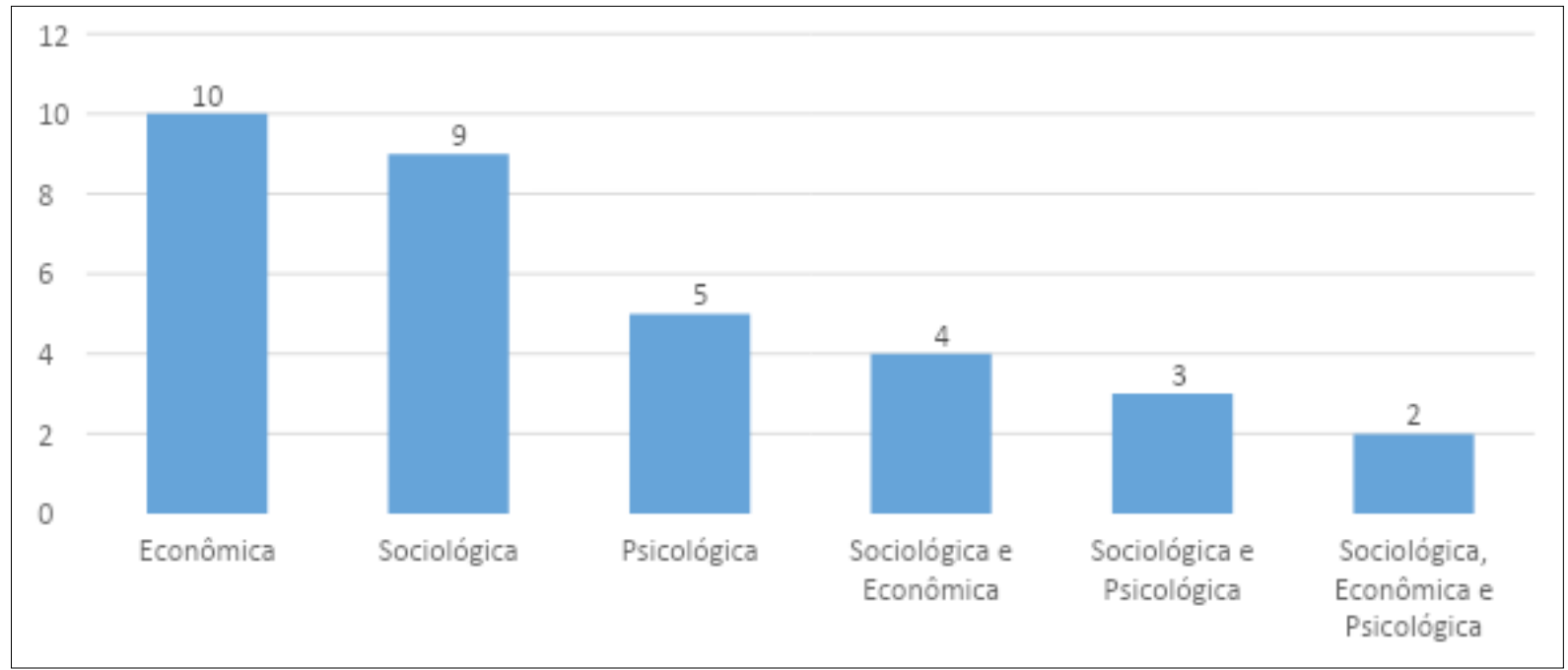

Fonte: Elaborada pelos autores.

Conforme a Figura 2, a maior parte das pesquisas internacionais sobre orçamento público adota uma perspectiva teórica econômica, o que vai ao encontro das recentes reformas na gestão pública com base nas empresas privadas que visam à melhoria do desempenho organizacional (Abu-Doleh \& Weir, 2007; Conway et al., 2016; Tanwir \& Chaudhry, 2016). Em seguida, destacam-se as pesquisas com enfoque sociológico, em especial com a adoção das Teorias de Contingência e Institucional, que buscam compreender como se formam e se modificam os processos orçamentários das organizações. Sendo assim, as próximas sub-seções vão tratar mais detalhadamente sobre as variáveis orçamentárias e não orçamentárias de cada perspectiva teórica adotada nos estudos orçamentários.

\subsubsection{Perspectiva teórica econômica}

Para os artigos que tiveram um enfoque econômico, a Tabela 3 descreve as variáveis estudadas. Destaca-se que a variável desempenho organizacional foi a que teve maior concentração. Todavia, considerando que essas variáveis não são isoladas entre si, já que alguns artigos, por exemplo, tratam de desempenho organizacional, mas de certa forma tratam também das características das práticas de orçamento, essa subseção vai descrever algumas características sobre os dez artigos encontrados que abordam a perspectiva econômica.

Tabela 3 - Variáveis da perspectiva econômica identificadas nos artigos

\begin{tabular}{|c|c|c|}
\hline & Variáveis econômicas & Artigos \\
\hline \multirow[b]{2}{*}{ Orçamentárias } & Orçamento participativo & 4 \\
\hline & $\begin{array}{l}\text { Características das práticas de orça- } \\
\text { mento }\end{array}$ & $21,30,31$ \\
\hline \multirow{2}{*}{ Não orçamentárias } & Resultado: desempenho organizacional & $12,17,23,27,28$ \\
\hline & Resultado: bem-estar individual & 20 \\
\hline
\end{tabular}

Considerando os períodos de austeridade fiscal, as recentes reformas na gestão pública têm encaminhado as instituições para uma centralização na tomada de decisões e na execução orçamentária, como no caso da Dinamarca (Kristiansen, 2018), em que a relação agente-principal foi reforçada em toda a cadeia de delegação por meio da aprovação 
dos orçamentos globais. Para melhorar esse controle orçamentário, Joharji e Willoughby (2014) também sugeriram a adoção de um orçamento global (top-down) e, ainda a avaliação dos programas por meio de indicadores de desempenho, já que a abordagem bottom-up (de baixo para cima) adotada na Arábia Saudita para execução orçamentária, por meio da solicitação de recursos financeiros pelas agências sem restrições macroeconômicas, resulta no aumento de assimetria da informação.

Por outro lado, Kan, Li e Tsai (2014) identificaram que a adoção de orçamento global no governo de Taiwan, em virtude de cortes orçamentários, não aumentou a intensidade dos tratamentos médicos aplicados, ou seja, não houve melhoria no bem-estar dos pacientes. Percebe-se que os estudos citam aspectos da Teoria da Agência, usada em estudos sociais para explicar a assimetria da informação existente nas organizações, em que os superiores detêm mais informações a nível macro e os subordinados detém mais informações a nível micro. Essa assimetria da informação traz incerteza à tomada de decisão e é percebida pelos administradores como uma ameaça à criação de valor.

A Teoria da Agência também esteve presente no estudo de Kim e Schachter (2013), ao identificarem a redução da assimetria da informação entre o principal e o agente por meio da aplicação do orçamento participativo ao nível local nos Estados Unidos e na Coréia do Sul. Neste estudo, os cidadãos foram considerados o polo principal e os funcionários foram considerados o polo agente. Já Ajibolade e Omobola (2017) citaram em seu estudo a Teoria Keynesiana, na qual os gastos públicos devem promover a economia, e concluíram que o atual sistema orçamentário da Nigéria falhou em promover significativo desenvolvimento da nação, sugerindo que seja dada maior transparência na gestão dos gastos públicos.

Outra opção para melhorar a eficiência e eficácia da gestão pública pode ser a adoção de práticas orçamentárias e financeiras recomendadas por organizações profissionais (Helpap, 2017). Entretanto, um estudo nos governos locais dos Estados Unidos demonstrou que algumas das práticas recomendadas não atingiram nem 50\% de taxa de adesão, o que afeta diretamente os resultados esperados (Helpap, 2017).

O orçamento com base no desempenho é outra estratégia que vem sendo adotada pelo setor público para aprimorar a sua gestão. Segundo Martí (2013), no Reino Unido, Austrália e Nova Zelândia são utilizadas medidas de produtos e resultados pretendidos e reais na alocação orçamentária, o que permite avaliações da eficácia dos programas públicos e contribui para a responsabilização por resultados. Todavia, por meio de um estudo nos Estados Unidos, Bischoff e Blaeschke (2016) alertaram que o orçamento com base no desempenho tem sido implementado de forma incompleta, pois suas informações não são usadas para alocar recursos, o que gera desperdício social, pois a reforma evoca custos adicionais sem oferecer qualquer benefício. Outra ressalva que deve ser feita em relação a adoção do orçamento baseado no desempenho é que ele estimula a eficiência e eficácia dos programas (nível micro), mas não é capaz de mudar permanentemente as prioridades nos gastos do governo em todas as funções sociais, em virtude de vários problemas como a falta de ligação clara entre os resultados de decisões orçamentárias e de desempenho, conforme estudo em onze países asiáticos efetuado por Srithongrung (2018).

Além disso, Sofyani (2018) testou empiricamente algumas variáveis relacionadas à implementação do orçamento baseado no desempenho com o sistema de avaliação de desempenho no governo local da Indonésia e descobriu que a falta de integração de informações entre esses dois sistemas prejudicou a eficácia do orçamento baseado no desempenho. Portanto, o orçamento com base no desempenho ainda é limitado a fatores organizacionais, como a falta de clareza das informações e a falta de vinculação das medidas de desempenho aos resultados finais.

\subsubsection{Perspectiva teórica sociológica}

Para os artigos que tiveram um enfoque sociológico, a Tabela 4 descreve as variáveis estudadas. A maioria dos artigos utilizou a Teoria Institucional para explicar as relações de poder que acontecem nas organizações públicas. Por isso, primeiro são apresentados esses artigos, e em seguida os demais.

Tabela 4 - Variáveis da perspectiva sociológica identificadas nos artigos

\begin{tabular}{l|l|l}
\hline & \multicolumn{1}{|c}{ Variáveis sociológicas } & Artigos \\
\hline Orçamentárias & $\begin{array}{l}\text { Teoria da Contingência: uso de } \\
\text { orçamentos operacionais para controle } \\
\text { gerencial. } \\
\text { Teoria institucional: processo } \\
\text { orçamentário. }\end{array}$ & 8 \\
& $\begin{array}{l}\text { Teoria institucional: valor simbólico da } \\
\text { contabilidade } \\
\text { Teoria institucional: mudança ambiental } \\
\text { e mudança organizacional. }\end{array}$ & $13,16,26,29,33$ \\
\hline
\end{tabular}

Mutiganda (2013) ampliou o quadro institucional de Burns e Scapens (2000) para o realismo crítico, demonstrando que em duas instituições públicas da Finlândia as práticas de accountability a partir de informações orçamentárias 
dependem de como as políticas institucionalizadas reduziram ou aumentaram as lacunas entre os domínios reais e empíricos dos atores organizacionais e da política de governança vigente. Dessa forma, conforme a Teoria Institucional, o orçamento é tido como um fenômeno socialmente construído, no qual as lógicas institucionais são legitimadas quando internalizadas pelos atores envolvidos.

Já Goetz e Patz (2016) demonstraram que a Comissão da União Européia respondeu às pressões sobre o orçamento europeu na recente crise financeira, por meio da centralização administrativa e medidas de proteção das rotinas do orçamento. Pressões institucionais também foram responsáveis por impossibilitar a implementação do orçamento-programa na Grécia, mesmo existindo a necessidade de focar na eficiência e eficácia dos gastos, considerando a crise financeira vigente (Cohen \& Karatzimas, 2014).

Cohen e Karatzimas (2014) embasaram sua pesquisa tanto por meio das lentes da teoria neoinstitucional, como da teoria da mudança organizacional e do modelo de dependência de recursos. Destacaram que mesmo que o orçamento-programa não tivesse sido abandonado ele teria dificuldade de sucesso por causa da resistência à mudança e da má difusão dos procedimentos contábeis, inerentes a um governo central burocrático (Cohen \& Karatzimas, 2014).

Bartocci, Grossi e Mauro (2019) também utilizaram a Teoria Neoinstitucional para estudar a evolução das lógicas institucionais no orçamento participativo em cinco municípios da Itália e identificaram que em um governo podem coexistir diferentes lógicas institucionais, como as lógicas gerenciais e de construção de comunidades que substituem ou coexistem com a lógica política tradicional. Ainda na Itália, Mauro, Cinquini e Grossi (2018) estudaram diferentes organizações públicas e mostraram que uma mesma prática, neste caso o orçamento baseado no desempenho, pode ou não ser internalizado e tornar-se institucionalizado, dependendo do alinhamento entre pressões externas e dinâmicas internas. A análise sublinhou como as pressões reguladoras por si só podem levar a comportamentos padronizados (isomorfismo), mas não necessariamente levar à internalização do orçamento com base no desempenho, pois as regras precisam ser normativamente endossadas e culturalmente suportadas (Mauro et al., 2018).

Destaca-se que a internalização das práticas da organização pelos atores institucionais é essencial para que as mesmas sejam institucionalizadas. Se o processo de internalização não for bem gerido, podem surgir comportamentos disfuncionais. Francesco (2016), por exemplo, explicou que os esforços sistêmicos de modernização orçamentária por meio da desconcentração podem ter falhado em fornecer flexibilidade orçamentária na Austrália, porque os atores institucionais internalizaram critérios da própria reforma que inibem a flexibilidade, o que impacta diretamente no comportamento da gestão orçamentária e financeira.

A gestão orçamentária assim, tanto pode moldar as lógicas institucionais inerentes às organizações públicas, como pode ser moldada por essas mesmas lógicas. Ao investigar um conselho urbano do Sri Lanka, Kuruppu (2016) descobriu que o orçamento participativo assumiu um papel simbólico, pois o presidente do conselho assumiu todo o controle do processo orçamentário e o orçamento foi dominado pela mesma dinâmica política.

Por outro lado, um controle orçamentário efetivo e rígido pode beneficiar as organizações públicas que estão enfrentando turbulências orçamentárias restritivas, como no caso dos municípios da Suécia (Johansson \& Siverbo, 2014). A Teoria da Contingência ajuda a explicar esses ajustes nos controles orçamentários de acordo com as mudanças ambientais.

Acerca dessas mudanças ambientais e organizacionais, Flink (2017) apresentou a teoria do equilíbrio pontuado (PET), em que Baumgartner e Jones (2010) relacionam o processo de política a fenômenos das ciências físicas, como terremotos, pois como estes, ocorrem de forma lenta e eventualmente levam a acontecimentos drásticos. Dessa forma, Flink (2017) concluiu acerca da direção da mudança orçamentária a partir de aspectos endógenos de distritos escolares do Texas (Estados Unidos), em que altos níveis de desempenho e baixos níveis de rotatividade de pessoal levam a mudanças incrementais.

\subsubsection{Perspectiva teórica psicológica}

Para os artigos que tiveram um enfoque psicológico, a Tabela 5 descreve as variáveis estudadas. As pesquisas com enfoque psicológico sobre o orçamento tiveram diferentes abordagens. Conway, Kiefer, Hartley e Briner (2014) pesquisaram sobre como os funcionários públicos de várias instituições do Reino Unido reagem à violação do contrato psicológico em relação ao comprometimento organizacional, após a mudança na organização derivada de medidas de austeridade por meio de fortes cortes orçamentários. Eles identificaram que não houve impacto sobre os colegas de trabalho e usuários do serviço público, porque os funcionários reagem contra o agente agressor, neste caso, a organização (Conway, Kiefer, Hartley \& Briner, 2014).

Tabela 5 - Variáveis da perspectiva psicológica identificadas nos artigos

\begin{tabular}{l|l|l}
\hline & \multicolumn{1}{|c|}{ Variáveis psicológicas } & Artigos \\
\hline \multirow{2}{*}{ Não orçamentárias } & $\begin{array}{l}\text { Estados mentais: atitudes, motivação, } \\
\text { satisfação, estresse } \\
\text { Comportamento: jogo }\end{array}$ & 1,2 \\
& Desempenho: individual e gerencial & 25,19 \\
\hline
\end{tabular}


Em relação ao Reino Unido, Kiefer, Hartley, Conway e Briner (2015) identificaram que o próprio anúncio de cortes orçamentários, antes de quaisquer mudanças serem efetuadas, já impactaram negativamente o bem-estar, as atitudes e os comportamentos dos funcionários. Além disso, diferentemente do impacto negativo sobre o bem-estar dos funcionários quando a mudança estava relacionada aos cortes orçamentários, houve impacto positivo sobre os funcionários quando as mudanças estavam relacionadas à inovação.

Becker et al. (2014) relataram sobre a interação dos contadores públicos da Alemanha com os programas ligados à Nova Gestão Pública e ao gerencialismo, em especial o orçamento com base no desempenho. Considerando que os contadores não reagiram de forma homogênea à reforma, percebeu-se que era necessário a simplificação do sistema Balanced Scorecard (BSC) para promover o interesse dos contadores (Becker et al., 2014).

Cohen (2015) apresentou uma análise, a partir da Teoria dos Jogos, da interação entre políticos e funcionários públicos em Israel com relação ao processo orçamentário nacional. Este estudo demonstrou como novas condições estruturais criaram novas regras que enfraqueceram a posição de negociação dos políticos, tornando os funcionários o lado dominante nesse jogo de poderes (Cohen, 2015).

Já Cuganesan (2017) demonstrou que a informação do orçamento de desempenho afeta a responsabilização da administração por meio dos arranjos de interesses de executivos, legisladores e funcionários da Austrália. Assim, os diferentes envolvidos interpretam a responsabilidade de diferentes maneiras e buscam persuadir os outros a seus pontos de vista, moldando a forma como os processos orçamentários são aprovados (Cuganesan, 2017).

\subsubsection{Perspectiva teórica sociológica e econômica}

Os artigos enquadrados na Tabela 6 apresentaram tanto variáveis sociológicas quanto variáveis econômicas. Ou seja, nota-se que alguns artigos já estão começando a integrar as perspectivas teóricas segundo Covaleski et al. (2007) para entender a dinâmica referente à elaboração, implementação e controle do orçamento público.

Tabela 6 - Variáveis das perspectivas sociológicas e econômicas identificadas nos artigos

\begin{tabular}{l|l|l|l}
\hline & \multicolumn{1}{|c|}{ Variáveis sociológicas } & \multicolumn{1}{c|}{ Variáveis econômicas } & Artigos \\
\hline $\begin{array}{l}\text { Orçamentárias e } \\
\text { não orçamentárias }\end{array}$ & $\begin{array}{l}\text { Teoria da Contingência: orçamento com } \\
\text { base no desempenho } \\
\text { Teoria institucional: processo } \\
\text { orçamentário. }\end{array}$ & $\begin{array}{l}\text { Resultado: desempenho organizacional } \\
\text { Carçamento }\end{array}$ & $7,15,22$ \\
\hline
\end{tabular}

Acerca das reformas na gestão pública, Hijal-Moghrabi (2017) analisou a adoção do orçamento baseado no desempenho nas maiores cidades dos Estados Unidos a partir da Teoria da Inovação e concluiu que embora as cidades tenham medidas de desempenho, os recursos não estão sendo alocados com base no desempenho. De forma semelhante, Ter Bogt et al. (2015) demonstraram que a complexidade do orçamento com base no desempenho de uma província holandesa parece questionar o pressuposto implícito da Nova Gestão Pública de racionalidade (econômica) na tomada de decisões, pois os resultados mostraram que o uso das informações de orçamento com base no desempenho em reuniões oficiais dos conselhos provinciais permanece limitado.

Bleyen, Klimovský, Bouckaert e Reichard (2017) também contribuíram com a incorporação de informações sobre desempenho nos orçamentos dos governos locais europeus, concluindo que a variação no uso dessas informações envolve os diferentes graus de implementação da reforma, a experiência com o orçamento baseado no desempenho e os arranjos institucionais vigentes. Gooneratne e Hoque (2016) desenvolveram em seu estudo de forma ainda mais clara, essa integração de aspectos econômicos (microprocessos) e sociológicos (Teoria Institucional), demonstrando que a institucionalização do sistema orçamentário em uma entidade híbrida (banco comercial estatal do Sri Lanka) é materializada em meio a: influências institucionais externas, deliberações de agentes organizacionais-chave e idiossincrasias na competitividade e complexidade do cenário comercial e organizacional.

\subsubsection{Perspectiva teórica sociológica e psicológica}

Os artigos enquadrados na Tabela 7 apresentaram tanto variáveis sociológicas quanto variáveis psicológicas, que conectam fatores exógenos (como pressões externas) a fatores endógenos (como comportamento dos indivíduos). 
Tabela 7 - Variáveis das perspectivas sociológicas e psicológicas identificadas nos artigos

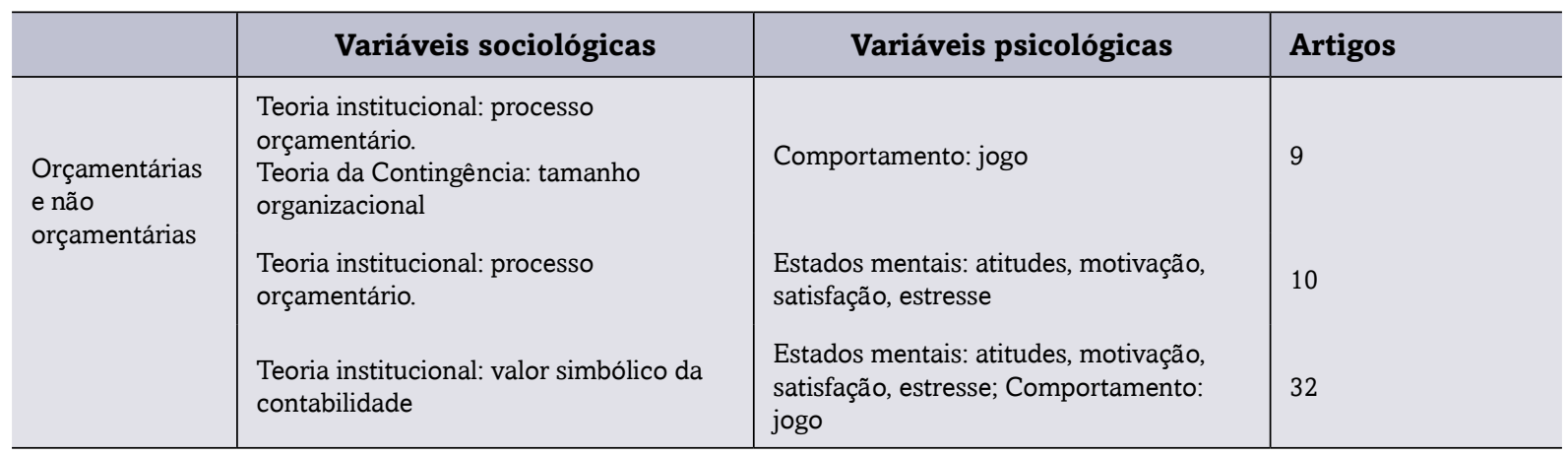

Grossi, Reichard e Ruggiero (2016) observaram em municípios da Alemanha e da Itália que o uso do orçamento com base nas informações de desempenho é bastante modesto, pois o foco ainda não é totalmente direcionado aos resultados (serviços prestados), fruto de diversos fatores contingenciais, como o foco tradicional de burocratas e políticos sobre os insumos e procedimentos. Ou seja, por mais que há pressão para que sejam utilizadas as informações do orçamento com base no desempenho, sua utilização efetiva é limitada pelo comportamento tradicional dos atores institucionais.

Ahrens e Ferry (2018) exploraram como uma organização pública de Israel de nível hierárquico inferior pode atuar como um empreendedor institucional, com base na Teoria Neoinstitucional. Esse estudo investiga a endogeneização das regras orçamentárias como um elemento importante do empreendedorismo institucional, que pode decorrer da evolução das emoções em jogo dos atores institucionais (memória cultural).

Mutiganda (2016) estudou os distritos hospitalares da Finlândia e identificou que o orçamento era uma ferramenta "cerimonial" de governança política, pois não houve relação direta entre os orçamentos e as práticas médicas. Nesse cenário os políticos influenciam o orçamento enquanto os médicos tentam responder à pressão política para cortar gastos. O raciocínio baseia-se na teoria da estruturação, em que quando um agente usa uma estrutura particular torna-se parte de seus traços em ações atuais e futuras (Giddens, 1984 como citado em Mutiganda, 2016).

\subsubsection{Perspectiva teórica sociológica, econômica e psicológica}

Observou-se que ainda são poucas as pesquisas que visam integrar as três perspectivas teóricas acerca do orçamento: econômica, sociológica e psicológica. Os dois artigos citados na Tabela 8, no entanto, podem ser considerados precursores na busca por um entendimento mais abrangente sobre as dinâmicas que regem o orçamento público.

Tabela 8 - Variáveis das perspectivas econômicas, sociológicas e psicológicas identificadas nos artigos

\begin{tabular}{l|l|l|l|l}
\hline & Variáveis econômicas & Variáveis sociológicas & Variáveis psicológicas & Artigos \\
\hline $\begin{array}{l}\text { Orçamentárias e } \\
\text { não orçamentárias }\end{array}$ & $\begin{array}{l}\text { Contratos baseados em } \\
\text { desempenho }\end{array}$ & $\begin{array}{l}\text { Teoria institucional: valor } \\
\text { simbólico da contabilidade } \\
\text { Teoria institucional: } \\
\text { processo orçamentário }\end{array}$ & Comportamento: jogo & 6 \\
\hline
\end{tabular}

Covaleski, Dirsmith e Weiss (2013) investigaram quais são os processos pelos quais os empresários institucionais de municípios da Suécia influenciam e aplicam as regras do jogo, expressa no novo regime de regulamentação do bem-estar orçamentário, tornando-os processos endógenos que transformam os próprios contextos organizacionais. Os autores identificaram que o orçamento facilitou a mudança de uma estratégia abrangente para incremental, por meio da flexibilidade necessária para transpor recursos de modo racional (sistema de entrega baseado no mercado e contratos com base em desempenho), garantindo a legitimidade dos arranjos organizacionais emergentes e os cortes orçamentários (Covaleski et al., 2013). Esse estudo amplia a visão do orçamento como um fenômeno multifacetado e socialmente construído (Merchant \& Van der Stede, 2006; Scapens \& Bromwich, 2001 como citado em Covaleski et al., 2013, p. 334), em que fatores sociológicos, psicológicos e econômicos estão interligados.

O estudo de Kim e Eom (2016) forneceu evidências de que os distritos escolares de Nova Jersey (Estados Unidos) com conselhos escolares eleitos são mais efetivos na redução dos gastos públicos do que aqueles cujos conselhos escolares são nomeados, pois estes conselhos elaboram o orçamento de acordo com os interesses dos administradores escolares. O embasamento teórico desse estudo foi composto por uma nova abordagem da Teoria Institucional, que englobou a Teoria dos Custos de Transação e a Teoria da Agência (Kim \& Eom, 2016). 


\section{CONSIDERAÇÕES FINAIS}

O presente estudo teve como objetivo identificar as características dos artigos científicos internacionais e classificar esses estudos de acordo com as perspectivas teóricas segundo Covaleski et al. (2007): econômica, psicológica e sociológica. $\mathrm{O}$ alcance do objetivo foi alcançado por meio de uma revisão sistemática da literatura, a partir da ferramenta Proknow-C, pela qual foi possivel coletar e filtrar os artigos sobre o tema. O portfólio final foi composto por 33 artigos.

Primeiramente, foi possível verificar que as pesquisas sobre o orçamento público estão se desenvolvendo em diferentes partes do mundo, sem qualquer concentração local. Em seguida, em relação às metodologias aplicadas, identificou-se predominância das abordagens qualitativas, efetuada por meio da análise documental e entrevistas com os envolvidos.

Em seguida, foi apresentada a classificação dos 33 artigos de acordo com as perspectivas teóricas segundo Covaleski et al. (2007). A perspectiva econômica se destacou, tendo em vista as recentes reformas no setor público, em busca da melhoria de seu desempenho (Abu-Doleh \& Weir, 2007; Conway, Fu, Monks, Alfes \& Bailey, 2016; Tanwir \& Chaudhry, 2016), derivada em muitos casos de medidas de austeridade fiscal, como fortes cortes orçamentários. Nesse sentido, a busca por um maior controle sobre o orçamento tem levado as instituições a centralizarem a execução orçamentária, reforçando a relação agente-principal (Kristiansen, 2018). Assim, a variável "desempenho" se destacou consideravelmente, uma vez que o setor público precisa demonstrar à sociedade que está gerindo os recursos públicos com eficiência.

A perspectiva sociológica também se destacou, tanto de forma isolada, quanto de forma integrada às perspectivas econômicas e psicológicas. À luz da Teoria Institucional e Neoinstitucional, os artigos estruturaram suas pesquisas com enfoque sociológico, reconhecendo que o orçamento é um fenômeno socialmente construído, no qual as lógicas institucionais são legitimadas quando internalizadas pelos atores envolvidos. Logo, a variável que se destacou foi o "processo orçamentário", isto é, o orçamento visto como um instrumento dinâmico, capaz de ser usado pelos grupos sociais como um meio para alcançar seus interesses.

A perspectiva psicológica tratou, principalmente, das variáveis "atitudes e comportamento" dos atores institucionais em relação às mudanças organizacionais, a exemplo do estudo de Kiefer et al. (2015), que descreveu o impacto negativo dos cortes orçamentários sob o bem-estar dos funcionários. Assim, essa perspectiva ressalta que o comportamento dos indivíduos deriva, em parte, das variáveis organizacionais a que são expostos. Num orçamento participativo, por exemplo, em que as vozes dos indivíduos possam ser consideradas, haverá menor resistência acerca de eventuais alterações que precisam acontecer na organização.

Por fim, foram encontrados nove artigos que integraram variáveis orçamentárias e não-orçamentárias de mais de uma perspectiva teórica. Destacando-se, especialmente, os artigos de Covaleski et al. (2013) e Kim e Eom (2016), que buscaram integrar as perspectivas econômica, sociológica e psicológica, ampliando o entendimento de que o orçamento público está ligado a diferentes fatores exógenos e endógenos da organização, como preferências dos atores, jogos de poder, comportamentos individuais, pressões externas. Independentemente de que tipo de orçamento o setor público adote (ex: orçamento com base no desempenho, orçamento participativo, orçamento-programa), foi possível reafirmar o entendimento de Covaleski et al. (2007), de que o sistema orçamentário será melhor compreendido a partir da integração das perspectivas econômica, sociológica e psicológica.

Assim, para que o orçamento público consiga acompanhar as mudanças de acordo com o contexto das organizações, ao mesmo tempo em que continua impondo regras na aplicação de recursos públicos é necessário entender o impacto do orçamento sobre a organização como um todo. Desta forma, a compreensão do orçamento público com base nessas diferentes perspectivas pode ampliar a visão dos gestores acerca do papel do orçamento sobre a organização, não como um instrumento estático, mas como um instrumento capaz de mudar as relações organizacionais e capaz de ser moldado a partir das atitudes dos atores envolvidos.

As delimitações desta pesquisa foram: (i) utilização de artigos selecionados a partir da base de dados Web of Science, em língua inglesa e acessivieis na internet; (ii) utilização de um comando de busca específico; (iii) análise das características dos artigos referente à localização dos estudos e à metodologia aplicada; e (iv) classificação dos artigos segundo às perspectivas teóricas de Covaleski et al. (2007) com base no julgamento e na interpretação dos autores.

Como sugestões para futuras pesquisas, apontam-se: (i) busca de artigos em outras bases de dados; (ii) desenvolvimento da análise de outras características; (iii) desenvolvimento da etapa do instrumento Proknow-C referente à análise sistêmica; e (iv) desenvolvimento de pesquisas que integrem as perspectivas econômicas, sociológicas e psicológicas.

\section{REFERÊNCIAS}

Abu-Doleh, J., \& Weir, D. (2007). Dimensions of performance appraisal systems in Jordanian private and public organizations. The International Journal of Human Resource Management, 18(1), 75-84.

Ahrens, T., \& Ferry, L. (2018). Institutional entrepreneurship, practice memory, and cultural memory: Choice and creativity in the pursuit of endogenous change of local authority budgeting. Management Accounting Research, 38, 12-21. [10]

Ajibolade, S. O., \& Oboh, C. S. (2017). A critical examination of government budgeting and public funds management in Nigeria. International Journal of Public Leadership, 13(4), 218-242. [23] 
Becker, S. D., Jagalla, T., \& Skærbæk, P. (2014). The translation of accrual accounting and budgeting and the reconfiguration of public sector accountants' identities. Critical Perspectives on Accounting, 25(4-5), 324-338. [5]

Bleyen, P., Klimovský, D., Bouckaert, G., \& Reichard, C. (2017). Linking budgeting to results? Evidence about performance budgets in European municipalities based on a comparative analytical model. Public Management Review, 19(7), 932-953. [15]

Bartocci, L., Grossi, G., \& Mauro, S. G. (2019). Towards a hybrid logic of participatory budgeting. International Journal of Public Sector Management, 32(1), 65-79. [26]

Bischoff, I., \& Blaeschke, F. (2016). Performance budgeting: Incentives and social waste from window dressing. Journal of Public Administration Research and Theory, 26(2), 344-358. [17]

Bradbury, J. C., \& Crain, W. M. (2001). Legislative organization and government spending: cross-country evidence. Journal of public Economics, 82(3), 309-325.

Burns, J., \& Scapens, R. W. (2000). Conceptualizing management accounting change: an institutional framework. Management accounting research, 11(1), 3-25.

Cohen, N. (2015). Bargaining and informal interactions in the national budget: A game theory analysis of the Israeli case. International Review of Administrative Sciences, 81(1), 58-78. [19]

Cohen, S., \& Karatzimas, S. (2014). Reporting performance information in the public sector: the moral behind the (non) application of program budgeting in Greece. International Review of Administrative Sciences, 80(3), 619-636. [16]

Conway, E., Fu, N., Monks, K., Alfes, K., \& Bailey, C. (2016). Demands or resources? The relationship between HR practices, employee engagement, and emotional exhaustion within a hybrid model of employment relations. Human Resource Management, 55(5), 901-917.

Conway, N., Kiefer, T., Hartley, J., \& Briner, R. B. (2014). Doing more with less? Employee reactions to psychological contract breach via target similarity or spillover during public sector organizational change. British Journal of Management, 25(4), 737-754. [1]

Covaleski, M. A., Dirsmith, M. W., \& Weiss, J. M. (2013). The social construction, challenge and transformation of a budgetary regime: The endogenization of welfare regulation by institutional entrepreneurs. Accounting, Organizations and Society, 38(5), 333-364. [6]

Covaleski, M. A., Evans III, J. H., Luft, J. L., \& Shields, M. D. (2007). Budgeting research: three theoretical perspectives and criteria for selective integration. Handbooks of management accounting research, 2(1), 587-624.

Cuganesan, S. (2017). The design of performance budgeting processes and managerial accountability relationships. Public Management Review, 19(7), 954-971. [25]

Flink, C. M. (2017). Rethinking punctuated equilibrium theory: A public administration approach to budgetary changes. Policy Studies Journal, 45(1), 101-120. [14]

Francesco, M. D. (2016). Rules and Flexibility in Public Budgeting: The Case of Budget Modernisation in Australia. Australian Journal of Public Administration, 75(2), 236-248. [33]

Goetz, K. H., \& Patz, R. (2016). Pressured budgets and the European Commission: towards a more centralized EU budget administration? Journal of European Public Policy, 23(7), 1038-1056. [11]

Gooneratne, T. N., \& Hoque, Z. (2016). Institutions, agency and the institutionalization of budgetary control in a hybrid state-owned entity. Critical Perspectives on Accounting, 36, 58-70. [18]

Grossi, G., Reichard, C., \& Ruggiero, P. (2016). Appropriateness and use of performance information in the budgeting process: Some experiences from German and Italian municipalities. Public Performance \& Management Review, 39(3), 581-606. [9]

Helpap, D. J. (2017). Guiding the Public Sector: Assessing the Use of Recommended Practices in the Budgeting Process. International Journal of Public Administration, 40(7), 559-574. [31]

Hijal-Moghrabi, I. (2017). The current practice of performance-based budgeting in the largest US cities: An innovation theory perspective. Public Performance \& Management Review, 40(4), 652-675. [22]

Hyvönen, T., Järvinen, J., Pellinen, J., \& Rahko, T. (2009). Institutional logics, ICT and stability of management accounting. European Accounting Review, 18(2), 241-275.

Johansson, T., \& Siverbo, S. (2014). The appropriateness of tight budget control in public sector organizations facing budget turbulence. Management Accounting Research, 25(4), 271-283. [8]

Joharji, G., \& Willoughby, J. (2014). The Saudi Arabian budgeting system: an institutional assessment. Public Administration and Development, 34(1), 63-80. [21]

Kan, K., Li, S. F., \& Tsai, W. D. (2014). The impact of global budgeting on treatment intensity and outcomes. International journal of health care finance and economics, 14(4), 311-337. [20]

Kiefer, T., Hartley, J., Conway, N., \& Briner, R. B. (2014). Feeling the squeeze: Public employees' experiences of cutback-and innovation-related organizational changes following a national announcement of budget reductions. Journal of Public Administration Research and Theory, 25(4), 1279-1305. [2]

Kim, S., \& Eom, T. H. (2016). A panel data analysis of the impacts of institutional differences on local governments' budgetary decisions. International Review of Administrative Sciences, 82(3), 559-579. [24]

Kim, S., \& Schachter, H. L. (2013). Citizen participation in the budget process and local government accountability: Case studies of organizational learning from the United States and South Korea. Public Performance \& Management Review, 36(3), 456-471. [4] 
Kristiansen, M. B. (2018). Budget reforms in times of austerity: A centralization cascade in Danish central government? Public Budgeting \& Finance, 38(3), 58-75. [30]

Kuruppu, C., Adhikari, P., Gunarathna, V., Ambalangodage, D., Perera, P., \& Karunarathna, C. (2016). Participatory budgeting in a Sri Lankan urban council: A practice of power and domination. Critical Perspectives on Accounting, 41, 1-17. [13]

Leite, R. M., Cherobim, A. P. M. S., Silva, H. D. F. N., \& Bufrem, L. S. (2008). Orçamento empresarial: levantamento da produção científica no período de 1995 a 2006. Revista Contabilidade \& Finanças, 19(47), 56-72.

Lunkes, R. J., Feliu, V. M. R., \& Rosa, F. S. (2011). Pesquisa sobre o orçamento na Espanha: um estudo bibliométrico das publicações em contabilidade. Revista Universo Contábil, 7(3), 112-132.

Martí, C. (2013). Performance budgeting and accrual budgeting: A study of the United Kingdom, Australia, and New Zealand. Public Performance \& Management Review, 37(1), 33-58. [12]

Mauro, S. G., Cinquini, L., \& Grossi, G. (2018). External pressures and internal dynamics in the institutionalization of performance-based budgeting: an endless process? Public Performance \& Management Review, 41(2), 224-252. [29]

Mutiganda, J. C. (2013). Budgetary governance and accountability in public sector organisations: An institutional and critical realism approach. Critical Perspectives on Accounting, 24(7-8), 518-531. [3]

Mutiganda, J. C. (2016). How do politicians shape and use budgets to govern public sector organizations? A position-practice approach. Public Money \& Management, 36(7), 491-498. [32]

Richardson, R. J. (2008). Pesquisa social: métodos e técnicas. São Paulo: Atlas.

Silva, M. Z., \& Lavarda, C. E. (2014). Orçamento empresarial: estudo comparativo entre publicações nacionais e internacionais. Revista Base (Administração e Contabilidade) da UNISINOS, 11(3), 179-192.

Sofyani, H. (2018). Does Performance-Based Budgeting Have a Correlation with Performance Measurement System? Evidence from Local Government in Indonesia. Foundations of Management, 10(1), 163-176. [28]

Srithongrung, A. (2018). An evaluation of performance-based budget reform in Asian countries. International Journal of Public Administration, 41(4), 257-267. [27]

Tanwir, M., \& Chaudhry, A. (2016). Reforming a broken system: a new performance evaluation system for Pakistan civil servants. Pakistan Development Review, 55(1), 49.

Ter Bogt, H. J., Van Helden, G. J., \& Van Der Kolk, B. (2015). Challenging the NPM ideas about performance management: Selectivity and differentiation in outcome-oriented performance budgeting. Financial Accountability \& Management, 31(3), 287-315. [7]

Valmorbida, S. M. I., \& Ensslin, S. R. (2017). Performance Evaluation of University Rankings: Literature Review and guidelines for future research. International Journal of Business Innovation and Research, 14(4), 479-501. 